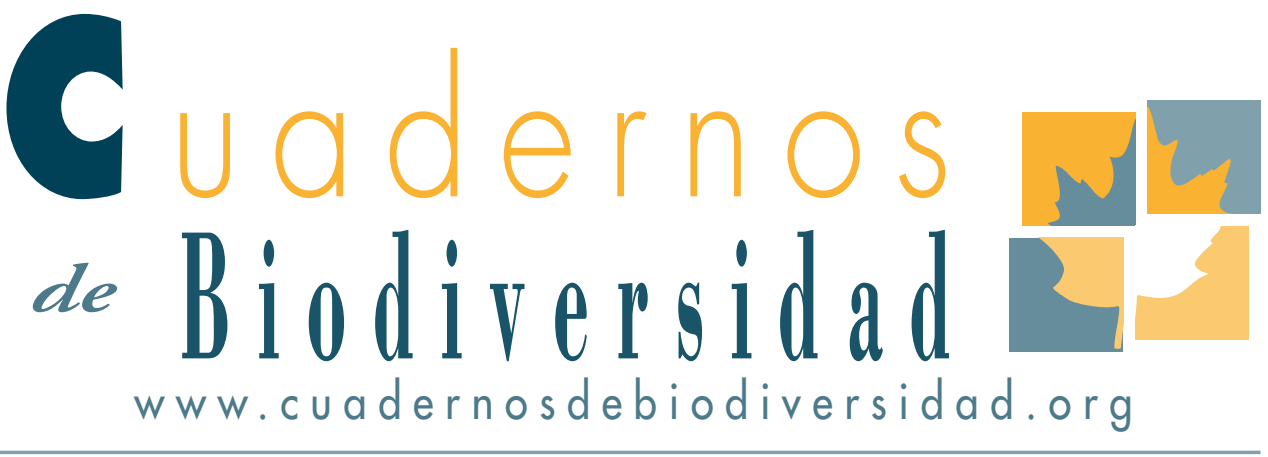

\title{
Conservación y distribución de las accesiones del Banco de Germoplasma de la Flora Silvestre Valenciana en la colección CIEF
}

\section{P. P. Ferrer-Gallego ${ }^{1,2}$, I. Ferrando ${ }^{1,2}$, F. Albert ${ }^{1,2}$, V. Martínez ${ }^{1,2}$, M. C. Escribä ${ }^{1,2}$, A. J. Navarro ${ }^{1,2}$ \& E. Laguna ${ }^{1}$}

i Servicio de Vida Silvestre. Generalitat Valenciana. CiEF (Centro para la Investigación y Experimentación Forestal), Generalitat Valenciana, Av. Comarques del País Valencià, i i 4, E-46930, Quart de Poblet, Valencia, España.

2 VAERSA. Avda. Cortes Valencianas, n²0. 460 i 5 , Valencia.

Datos DE CONTACTO: FLORA.CiEF@GMAIL.COM

\section{ABSTRACT}

The Germplasm Bank of Valencia Wild Flora is a network of collections for conservation of taxa listed in Valencia Catalog Endangered Species of Flora (VCESF). CIEF germplasm collection belongs to Generalitat Valenciana's Forest Research and Experimentation Centre, and in addition to forest species, seeds of singular plants of VCESF are also preserved by the Wildlife Service team. From 1990 until now, in bank's installations is preserved 2447 lots from 1445 species. It is conserved germplasm from $92 \%$ of the Endangered species and $94 \%$ as Vulnerable species according to VCESF. These data indicate that $67,6 \%$ of monitoring populations currently known for species listed as Endangered are preserved in the collection and 50\% in the case of Vulnerable species. High-density areas of harvesting are the north and northwest of Alicante (dianic area and "Sierra de Aitana"), the southeast quadrant of Valencia (setabense zone), the Valencian coast ("El Saler", "Sagunto", etc.), and Castellón ("Islas Columbretes", "Macizo del Penyagolosa”, "Cabanes", "Peñiscola"). The aim to short-medium term is to keep up at least one accession of each monitoring population from VCESF species capable to be conserved in a genebank. 
Conservation ex situ, georeferenced database, endemism, endangered species, seeds, Valencian Community.

\section{INTRODUCCIÓN}

El Banco de Germoplasma de la Flora Silvestre Valenciana (BGFSV) es una red de instalaciones que conservan material vegetal de reproducción (semillas, colecciones de planta viva, y otro tipo de propágulos) de especies de la flora presente en la Comunidad Valenciana (España), dando prioridad a los táxones protegidos y listados en el Catálogo Valenciano de Especies de Flora Amenazadas (según Decreto 70/2009 y Orden 6/2013) -en adelante CVEFA, o simplemente 'Catálogo'- , a los protegidos de categorías inferiores de la misma norma -especies Protegidas no Catalogadas, y especies Vigiladas- y a los endémicos exclusivos o iberolevantinos presentes en el territorio valenciano, entre otros muchos (Aguilella et al., 2009).

Dicha red la integran las colecciones sitas en el banco de germoplasma del Jardí Botànic de la Universitat de València, donde reside la sede central y la principal unidad de conservación a largo plazo, y en las instalaciones del Centro para la Investigación y Experimentación Forestal de la Generalitat Valenciana (CIEF) y el Centro de Investigación Piscícola El Palmar, donde adquieren más importancia las colecciones de semillas y de planta viva para la producción a corto o medio plazo (FerrerGallego et al., 2013a). Además, complementan estas colecciones el Banc de Llavors Forestals de la Comunitat Valenciana, adscrito al CIEF y dedicado a la conservación y producción de especies autóctonas arbóreas y arbustivas, desarrollando su principal actividad en el marco del programa ECOGEN (Prada et al., 2012).

La actividad del BGFSV se centra principalmente en la conservación y estudio la flora valenciana, según la citada normativa autonómica y de acuerdo con lo establecido en la Ley 42/2007 y la Estrategia Mundial para la Conservación de las Especies Vegetales 2011-2020 (decisión X/17 de la Conferencia de las Partes) del Convenio de Naciones Unidas sobre Diversidad Biológica (CDB) y particularmente para dar respuesta al compromiso de España en la Estrategia Española de Conservación Vegetal 2013-2020 enmarcada en la Estrategia Global de Conservación de Plantas del CDB.

En la actualidad, el BGFSV se muestra como una herramienta de gran importancia para la conservación de la flora valenciana, siendo una colección en constante ampliación, en lo que se refiere fundamentalmente al número de lotes y accesiones que procesa y conserva, así como al estudio de la calidad del material conservado y el número de accesiones que son enviadas a otros centros de conservación y/o investigación vegetal (Laguna et al., 2012; FerrerGallego et al., 2013b).

Uno de los retos más importantes que afronta la gestión de la biodiversidad es la disponibilidad de datos abundantes y de calidad de la distribución espacio-temporal, tanto de las especies como de sus hábitats (Boakes et al,, 2010). Cuanto mayor es el volumen de datos mejor se pueden sin duda detectar y evaluar tendencias en los sistemas naturales que no se manifiestan a partir de observaciones más restringidas (Uribe \& Prieto, 2014). Se trata de percepciones incubadas en la propia masa de datos cuando ésta adquiere un volumen crítico (Kelling et al., 2009). En respuesta a una demanda de información de calidad, se dispone de un número creciente de aplicaciones y recursos analíticos que hacen frente a complejos conjuntos de datos multidisciplinares (Frew \& Dozier, 2012). Así, los Sistemas de Información Geográfica (SIG) han modificado sustancialmente las metodologías de trabajo en conservación en los últimos años, resultando actualmente una herramienta habitual para el estudio de datos poblacionales, representación cartográfica y diferentes cálculos, así como para la toma de decisiones en la gestión práctica de las especies y el territorio. Iniciativas como GBIF (Global Biodiversity Information Facility, http://www.gbif. es/), a pesar de sus limitaciones (Beck et al., 2013) son de gran importancia para concentrar fuentes de información así como para suscitar el interés por el empleo de estándares de gestión y de distribución 
de contenidos de biodiversidad. Sin embargo, el uso de estos métodos y aplicaciones para obtener información de material vegetal vivo conservado ex situ apenas ha sido desarrollado, y en general se carece de trabajos de referencia en el ámbito valenciano y también nacional en esta materia.

La cantidad de accesiones conservadas en la sección del CIEF del Banco de Germoplasma de Flora Silvestre Valenciana, que han sido progresivamente recolectadas y procesadas entre 1995 y 2014 a lo largo de todo el territorio valenciano (véase Ferrer-Gallego et al., 2013b), permite plantear un análisis SIG de la información que georreferencia las accesiones de germoplasma almacenada en la base de datos, incluyendo: 1) la confección de mapas de densidad de recolección; 2) estimación de áreas con mayor concentración de esfuerzo, y relación de dichos esfuerzos con la distribución de las especies amenazadas; 3) localización de áreas y poblaciones con carencias en la recolección (áreas 'gap'); 4) estimación de la calidad del germoplasma en las unidades de seguimiento -parcelas de monitoreo regular en campo- de las especies más amenazadas, así como otros objetivos.

\section{MATERIAL Y MÉTODOS}

Las poblaciones y núcleos poblacionales individualizados de las especies del Catálogo Valenciano de Especies de Flora Amenazadas (CVEFA) son consideradas como unidades de seguimiento (US), es decir, unidades operacionales para su estudio, recolección de semillas y seguimiento demográfico, así como para los trabajos de restitución, entre otras acciones de conservación activa in situ (véase Bañares, 2003; Iriondo, 2011). Estas unidades de seguimiento son caracterizadas y registradas en el Banco de Datos de Biodiversidad de la Comunidad Valenciana (http://bdb.cth.gva.es/).

Las accesiones recolectadas son caracterizadas, catalogadas y registradas en una base de datos para el control de la trazabilidad del germoplasma, desde que entran en la colección hasta que salen en forma de planta para los trabajos de restitución en campo, metodología basada en lo indicado por Bacchetta et al. (2008) y fundamentada en determinados trabajos básicos (Falk, 1987; Cohen et al., 1991; Hamilton, 1994; Iriondo, 2001; Linington \& Pritchard, 2001; IPGRI/FAO/FLD, 2004), y que en concreto para la flora valenciana ya fuera expuesta por Ferrer-Gallego et al. (2013a).

La información utilizada en este análisis SIG de las accesiones del Banco de germoplasma, son las coordenadas U.T.M. (Universal Transversal Mercator) de $10 \mathrm{~km}$ de lado, que georreferencian las recolecciones (semillas, propágulos, y cualquier otro material vegetal de reproducción) y colecciones vegetales de planta viva conservadas en la colección CIEF del BGFSV.

Para estimar coordenadas U.T.M., en el caso de recolecciones antiguas, se ha efectuado una georreferenciación retrospectiva, basada en asignar coordenadas centroides al punto de recolección utilizando como herramientas el nombre del paraje natural señalado por el recolector en la ficha de la accesión y la búsqueda de esta toponimia en el visor web cartográfico de los montes gestionados por la Consellería de Infraestructuras, Territorio y Medio Ambiente (http://cartoweb.cma.gva.es/visor/).

El análisis y representación cartográfica de la información se ha realizado con el software libre gvSIG (versión 1.12 .0 final).

\section{RESULTADOS Y DISCUSIÓN}

Desde el año 1990 hasta la actualidad el número de lotes confeccionados que conservan germoplasma de la flora silvestre valenciana en la colección CIEF del BGFSV es 2447, de un total de 1445 especies (Tabla 1). Durante este tiempo, el número de lotes elaborados ha aumentado progresivamente, aunque fue en 2011 cuando se confeccionó un mayor número de lotes, muy probablemente como efecto de la publicación de la normativa por la que se crea el Catálogo Valenciano de Especies de Flora Amenazada (Decreto 70/2009), al igual que se observó en las pruebas de germinación de semillas del mismo 
banco de germoplasma (Laguna et al., 2012). En este sentido, respecto al número de especies que han sido recolectadas, el período con mayor intensidad recolectora se sitúa entre 2006 y 2008, disminuyendo sensiblemente a partir de 2009 , consecuencia también del efecto de la publicación de esta normativa, ya que la concentración de esfuerzos y recursos ha estado dirigida hacia un aumento del número de semillas de las poblaciones de las especies listadas en el citado decreto (Tabla 1).
En la actualidad, de las 35 especies catalogadas como En Peligro en el CVEFA, se conserva germoplasma de 26 táxones, en un total de 373 lotes, mientras que de las 50 especies catalogadas como Vulnerables, se conserva germoplasma de 33 de ellas, repartidas en 327 lotes. Todos estos lotes están debidamente caracterizados y georreferenciados (Tablas 2 y 3). Por otra parte, la cantidad de lotes de las especies listadas en la categoría Protegidas no Catalogadas es de 417 y para las Vigiladas es

Tabla 1. Número de lotes confeccionados de las especies recolectadas a lo largo de los años 1990-2014. * Accesiones recolectadas y procesadas hasta julio de 2014.

\begin{tabular}{|c|c|c|c|c|}
\hline \multirow{2}{*}{ Años } & \multicolumn{2}{|c|}{ Total de lotes confeccionados } & \multicolumn{2}{|c|}{ Total de especies recolectadas } \\
\hline & Anual & Acumulado & Anual & Acumulado \\
\hline 1990 & 6 & 6 & 3 & 3 \\
\hline 1991 & 3 & 9 & 3 & 6 \\
\hline 1992 & 2 & 11 & 2 & 8 \\
\hline 1993 & 4 & 15 & 4 & 12 \\
\hline 1994 & 13 & 28 & 8 & 20 \\
\hline 1995 & 19 & 47 & 8 & 28 \\
\hline 1996 & 19 & 66 & 9 & 37 \\
\hline 1997 & 53 & 119 & 41 & 78 \\
\hline 1998 & 47 & 166 & 24 & 102 \\
\hline 1999 & 24 & 190 & 21 & 123 \\
\hline 2000 & 48 & 238 & 39 & 162 \\
\hline 2001 & 61 & 299 & 40 & 202 \\
\hline 2002 & 82 & 381 & 58 & 260 \\
\hline 2003 & 177 & 558 & 125 & 385 \\
\hline 2004 & 147 & 705 & 109 & 494 \\
\hline 2005 & 178 & 883 & 95 & 589 \\
\hline 2006 & 171 & 1054 & 108 & 697 \\
\hline 2007 & 260 & 1314 & 179 & 876 \\
\hline 2008 & 236 & 1550 & 153 & 1029 \\
\hline 2009 & 124 & 1674 & 83 & 1112 \\
\hline 2010 & 171 & 1845 & 98 & 1210 \\
\hline 2011 & 287 & 2132 & 81 & 1291 \\
\hline 2012 & 139 & 2271 & 72 & 1363 \\
\hline 2013 & 110 & 2381 & 78 & 1441 \\
\hline $2014^{*}$ & 66 & 2447 & 54 & 1495 \\
\hline
\end{tabular}


de 245 lotes, de los cuales el 73,14\% y el 63,27\%, respectivamente están georreferenciados. También, se conserva un total de 1085 lotes de 356 especies no protegidas, de los cuales se ha georreferenciado hasta el momento el 41,75\% (Tabla 5).

Entre las especies que cuentan con un mayor número de lotes conservados para las unidades de seguimiento conocidas en la actualidad de las especies del CVEFA, se encuentran las siguientes -se indican con asterisco las endémicas absolutas valencianas: Limonium mansanetianum* (13 lotes $=13$ unidades de seguimiento conservadas ex situ), Lupinus mariae-josephae* (9), Limonium dufourii* (8), Helianthemum caput-felis (6), Antirrhinum valentinum* (5), Garidella nigellastrum (5), Diplotaxis ibicensis (4). En estos casos, todos estos táxones cuentan en parte con un gran número de unidades de seguimiento, o puntuales poblaciones pero con un elevado número de individuos. Con 4 lotes o unidades de seguimiento conservadas ex situ aparecen Leucojum valentinum*, Medicago citrina, Parentucellia viscosa, Silene hifacensis y Teucrium lepicephalum*. Por el contrario, las especies para las que hasta el momento no se conserva semillas en el banco de ninguna de las unidades de seguimiento son: Euphrasia salisburgensis, Launaea lanifera, Dactylorhiza incarnata y Vitaliana primuliflora subsp. assoana, en parte debido a la dificultad de germinación de las mismas o la falta de semillas viables en las poblaciones naturales (Tabla 2). Como puede observarse, cara a la conservación, se prima especialmente el caso de los endemismos absolutos, que son especies sobre la administración valenciana de la que depende el banco de germoplasma y posee una máxima responsabilidad (véase Laguna, 2002).

Tabla 2. Unidades de seguimiento de las especies listadas en el CVEFA y representación de las mismas
en el banco de germoplasma del CIEF
\begin{tabular}{l|l|r|r|r} 
Taxon & Cat & No Unid. & Recol & Conservado (\%) \\
\hline Allium subvillosum & EP & 2 & 1 & 50,0 \\
\hline Aristolochia clematitis & EP & 1 & 1 & 100,0 \\
\hline Berberis hispanica subsp. hispanica & EP & 1 & 1 & 100,0 \\
\hline Boerhavia repens & EP & 1 & 1 & 100,0 \\
\hline Centaurea alpina & EP & 1 & 1 & 100,0 \\
\hline Cistus heterophyllus subsp carthaginensis & EP & 2 & 2 & 100,0 \\
\hline Corema album & EP & 1 & 1 & 100,0 \\
\hline Cotoneaster granatensis & EP & 2 & 2 & 100,0 \\
\hline Euphrasia salisburgensis & EP & 1 & 0 & 0,0 \\
\hline Frangula alnus s.a. & EP & 1 & 1 & 100,0 \\
\hline Halimium atriplicifolium & EP & 4 & 3 & 75,0 \\
\hline Launaea arborescens & EP & 4 & 3 & 75,0 \\
\hline Launaea lanifera & EP & 2 & 0 & 0,0 \\
\hline Leucanthemum arundanum & EP & 2 & 2 & 100,0 \\
\hline Limonium bellidifolium & EP & 3 & 2 & 66,7 \\
\hline Limonium dufourii & EP & 8 & 8 & 100,0 \\
\hline Limonium lobatum & EP & 1 & 1 & 100,0 \\
\hline Limonium perplexum & EP & 1 & 1 & 100,0 \\
\hline Narcissus perezlarae & EP & 6 & 1 & 16,7 \\
\hline Orchis papilionacea & EP & 8 & 2 & 25,0 \\
\hline Parentucellia viscosa & EP & 8 & 4 & 50,0 \\
\hline Reseda hookeri & EP & 1 & 1 & 100,0 \\
\hline & & & \\
\hline
\end{tabular}




\begin{tabular}{|c|c|c|c|c|}
\hline Reseda lanceolata & EP & 1 & 1 & 100,0 \\
\hline Rumex roseus & EP & 3 & 2 & 66,7 \\
\hline Silene cambessedesii & EP & 2 & 2 & 100,0 \\
\hline Silene hifacensis & EP & 4 & 4 & 100,0 \\
\hline Ajuga pyramidalis subsp. meonantha & VU & 3 & 1 & 33,3 \\
\hline Antirrhinum valentinum & VU & 17 & 5 & 29,4 \\
\hline Armeria fontqueri & VU & 2 & 2 & 100,0 \\
\hline Astragalus oxyglottis & VU & 3 & 2 & 66,7 \\
\hline Callipeltis cucullaria & $\mathrm{VU}$ & 5 & 3 & 60,0 \\
\hline Campanula mollis & VU & 1 & 1 & 100,0 \\
\hline Centaurea lagascae & VU & 2 & 2 & 100,0 \\
\hline Clematis cirrhosa & $\mathrm{VU}$ & 4 & 2 & 50,0 \\
\hline Dactylorhiza incarnata & VU & 6 & 0 & 0,0 \\
\hline Dianthus carthusianorum & VU & 1 & 1 & 100,0 \\
\hline Diplotaxis ibicensis & $\mathrm{VU}$ & 7 & 4 & 57,1 \\
\hline Euphorbia nevadensis subsp. nevadensis & VU & 2 & 1 & 50,0 \\
\hline Fumaria munbyi & VU & 1 & 1 & 100,0 \\
\hline Garidella nigellastrum & VU & 6 & 5 & 83,3 \\
\hline Genista umbellata & VU & 1 & 1 & 100,0 \\
\hline Halopeplis amplexicaulis & VU & 3 & 1 & 33,3 \\
\hline Helianthemum caput-felis & $\mathrm{VU}$ & 18 & 6 & 33,3 \\
\hline Kernera saxatilis subsp. boissieri & VU & 2 & 2 & 100,0 \\
\hline Leucojum valentinum & VU & 21 & 4 & 19,0 \\
\hline Limonium mansanetianum & $\mathrm{VU}$ & 13 & 13 & 100,0 \\
\hline Lupinus mariae-josephae & $\mathrm{VU}$ & 9 & 9 & 100,0 \\
\hline Maytenus senegalensis subsp. europaea & $\mathrm{VU}$ & 2 & 1 & 50,0 \\
\hline Medicago citrina & $\mathrm{VU}$ & 4 & 4 & 100,0 \\
\hline Notoceras bicorne & VU & 1 & 1 & 100,0 \\
\hline Odontites valentinus & VU & 4 & 3 & 75,0 \\
\hline Orchis conica & VU & 12 & 1 & 8,3 \\
\hline Ribes uva-crispa & VU & 2 & 1 & 50,0 \\
\hline Salsola soda & VU & 2 & 1 & 50,0 \\
\hline Solenopsis laurentia & VU & 2 & 2 & 100,0 \\
\hline Teucrium lepicephalum & $\mathrm{VU}$ & 10 & 4 & 40,0 \\
\hline Thymus ricardii subsp. vigoi & $\mathrm{VU}$ & 1 & 1 & 100,0 \\
\hline Thymus webbianus & VU & 3 & 1 & 33,3 \\
\hline Vitaliana primuliflora subsp. assoana & VU & 2 & 0 & 0,0 \\
\hline
\end{tabular}

Respecto a las unidades de seguimiento (US) de la flora catalogada como En Peligro de extinción, para 16 especies se conserva el $100 \%$ de las US en lotes de semillas, para 6 especies entre el 50 y 75\% de las US conocidas, y restan 2 especies para las que todavía no se conserva germoplasma de ninguna de sus US (Tabla 4). En total, para esta categoría, son 48 el número de US conservadas de un total de 24 especies (Tabla 3). Para la flora catalogada como Vulnerable, 13 especies cuentan con el 100\% de las US 
conservadas, y 10 especies entre el 50 y $75 \%$ de las US, de igual manera que para la categoría anterior, de 2 especies todavía no se conserva germoplasma de ninguna de sus US (Tabla 4). Así, para esta categoría, son 86 el número de US conservadas de un total de 31 especies (Tabla 3).

Tabla 3. Número de especies, unidades de seguimiento y su conservación en el banco de germoplasma para el CVEFA

\begin{tabular}{c|c|c|c|c} 
Categoría & $\begin{array}{c}\text { Número de } \\
\text { especies }\end{array}$ & $\begin{array}{c}\text { Unidades de } \\
\text { seguimiento }\end{array}$ & $\begin{array}{c}\text { Número de especies } \\
\text { conservadas }\end{array}$ & $\begin{array}{c}\text { Unidades de seguimiento } \\
\text { conservadas }\end{array}$ \\
\hline En Peligro & 26 & 71 & 24 & 48 \\
\hline Vulnerable & 33 & 172 & 31 & 86
\end{tabular}

Tabla 4. Rangos de unidades de seguimiento conservadas en accesiones en el banco de las especies catalogadas como En Peligro y Vulnerables

\begin{tabular}{c|c|c} 
Unidades de seguimiento conservadas & $\begin{array}{c}\text { No especies } \\
\text { En Peligro }\end{array}$ & No especies Vulnerables \\
\hline $\mathbf{1 0 0 \%}$ & 16 & 13 \\
\hline Entre $\mathbf{5 0}$ y $\mathbf{7 5 \%}$ & 6 & 10 \\
\hline Entre $\mathbf{2 5}$ y $\mathbf{5 0 \%}$ & 2 & 6 \\
\hline Menos de $\mathbf{2 5} \%$ & 0 & 2 \\
\hline 0\% & 2 & 2 \\
\hline TOTAL & $\mathbf{2 6}$ & $\mathbf{3 3}$
\end{tabular}

Tabla 5. Número de lotes georreferenciados según las categorías de amenaza de las especies.

\begin{tabular}{l|c|c|c|c}
\multicolumn{1}{c|}{ Categoría } & Especies & Lotes & $\begin{array}{c}\text { Lotes } \\
\text { georreferenciados }\end{array}$ & $\begin{array}{c}\text { Lotes georreferenciados } \\
(\%)\end{array}$ \\
\hline En Peligro & 26 & 373 & 373 & 100,00 \\
\hline Vulnerable & 33 & 327 & 327 & 100,00 \\
\hline Protegida no Catalogada & 54 & 417 & 305 & 73,14 \\
\hline Vigilada & 32 & 245 & 155 & 63,27 \\
\hline No protegida & 356 & 1085 & 453 & 41,75 \\
\hline Total & $\mathbf{5 0 1}$ & $\mathbf{2 4 4 7}$ & $\mathbf{1 6 1 3}$ & $\mathbf{6 5 , 9 2}$
\end{tabular}


La representación espacial del número de lotes conservados de las especies listadas en la CVEFA permite observar varias áreas de alta densidad de recolección, como por ejemplo el norte y noroeste de Alicante (zona diánica, y sierra de Aitana), el cuadrante suroriental de Valencia (zona setabense), varias cuadrículas de la costa valenciana (El Saler, Sagunto, etc.), y en Castellón además de la intensidad de recolección de las Islas Columbretes, poblaciones presentes en el Macizo del Penyagolosa (y otras zonas del interior) y varios puntos del litoral (Cabanes, Peñíscola, etc.) (Figuras 1 y 2). Un objetivo a corto-medio plazo es conservar al menos una accesión de cada unidad de seguimiento de las especies del CVEFA susceptibles de ser conservadas en el banco de germoplasma.

Las especies no abarcadas por el banco de germoplasma, de las que obviamente no se tienen accesiones georreferenciadas, corresponden o bien

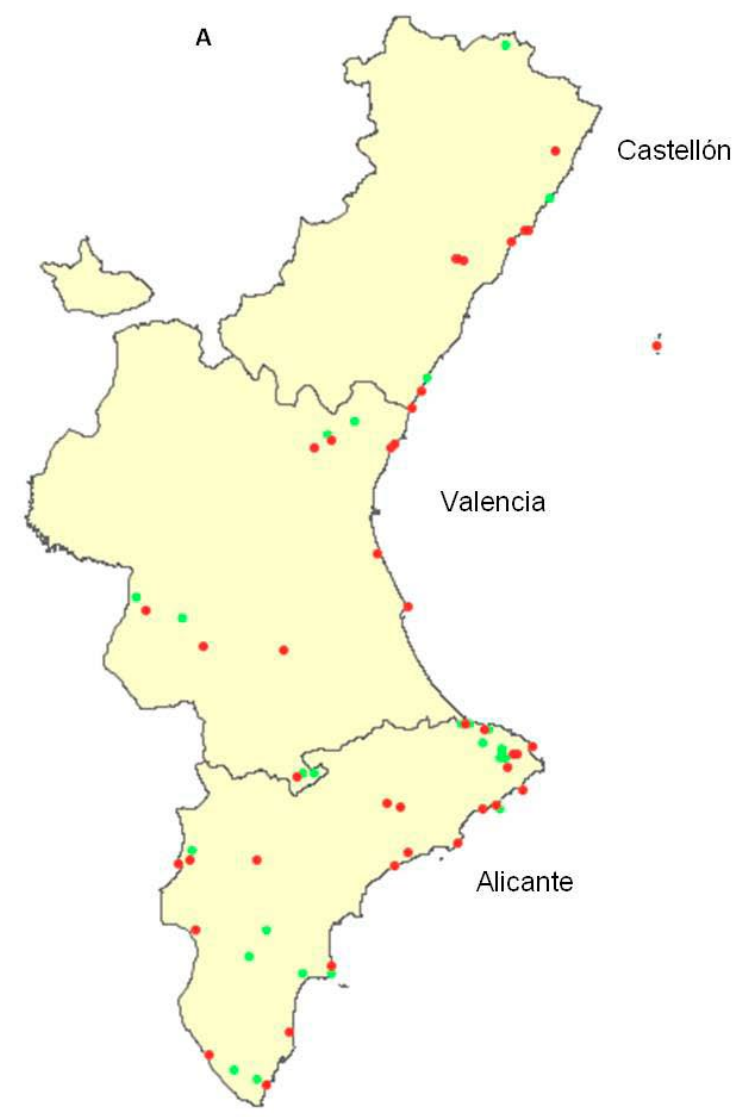

a táxones recalcitrantes que no se puede conservar en bancos de semillas (p. ej. Nymphaea alba y otras especies acuáticas), o bien a especies extintas o no reencontradas en el territorio valenciano.

La representación espacial de la calidad del germoplasma (viabilidad y germinabilidad de semillas) de las unidades de seguimiento de las especies de flora más amenazadas puede dar una idea básica y algunas respuestas a determinadas dinámicas de las poblaciones observadas. Así, para algunas unidades de seguimiento se ha constatado una baja calidad de su germoplasma, lo que puede estar directamente relacionado con el reducido reclutamiento de nuevos individuos en la población (Halimium atriplicifolium, Reseda hookeri, Solenopsis laurentia, Vitaliana primuliflora subsp. assoana). Por el contrario, viabilidades altas en algunas accesiones (por ejemplo de Limonium mansanetianum) explican parcialmente ciertas explosiones demográficas con un aumento sustancial del número de efectivos poblacionales.

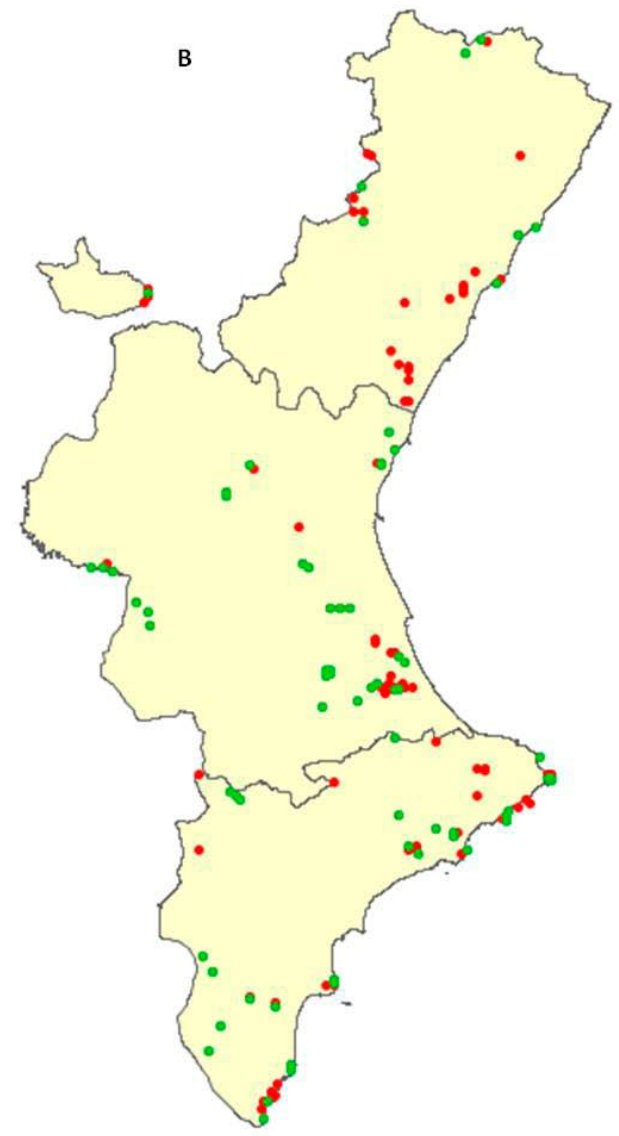

Fig. 1. Representación de las unidades de seguimiento de las especies catalogadas como "En Peligro" (A) y "Vulnerables" (B); en rojo las unidades de las que se cuenta con germoplasma conservado. 

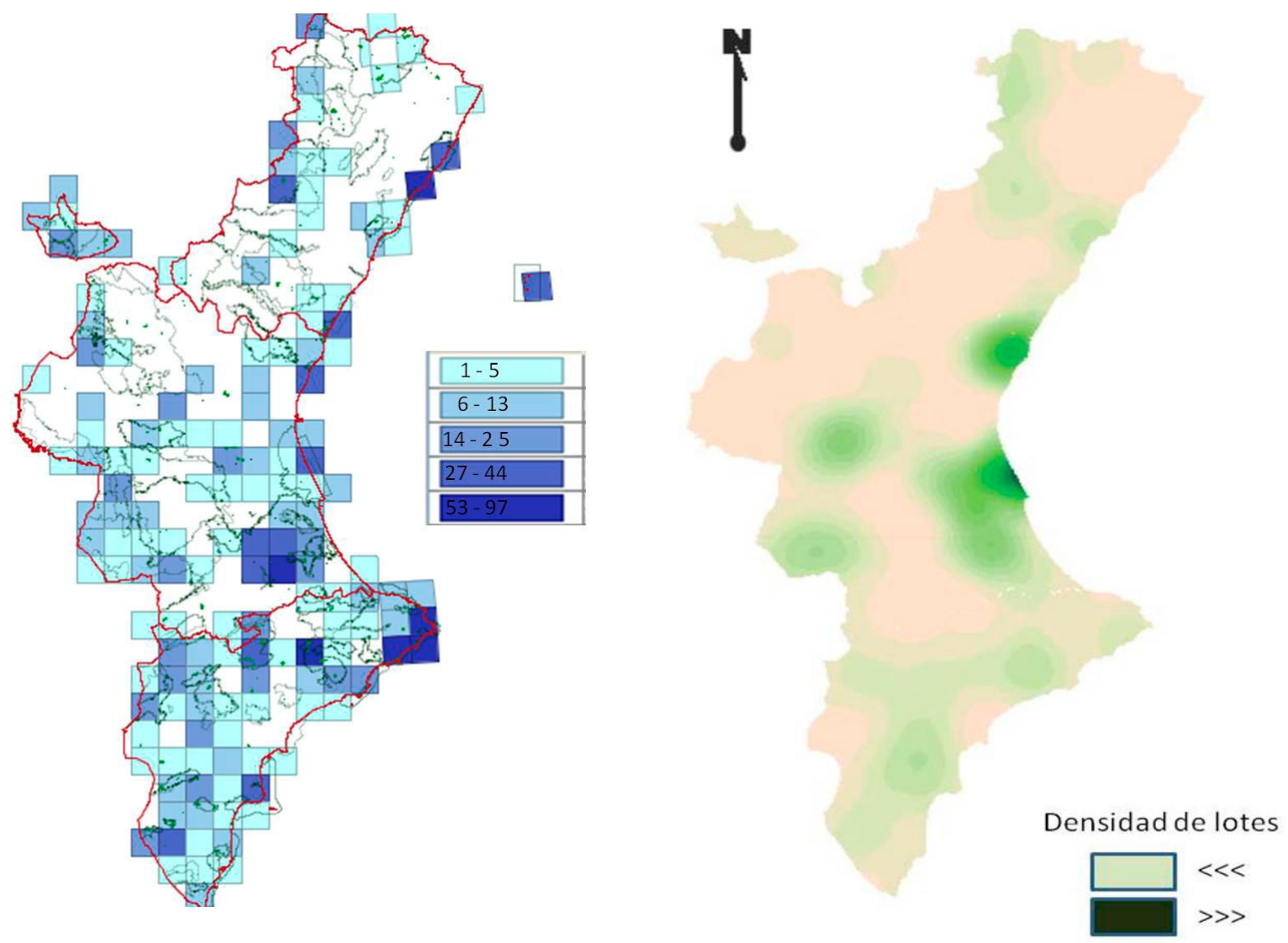

Fig. 2. Representación de la densidad de lotes por cuadrícula 10 x $10 \mathrm{~km}$ conservados en el banco de germoplasma del CIEF. Las líneas verdes indican las áreas correspondientes a espacios de la red Natura 2000 y microreservas de flora (a la derecha representación del número de lotes/área mediante isolíneas de densidad).

\section{CONCLUSIONES}

Para el $92 \%$ de las especies catalogadas como En Peligro y para el $94 \%$ de las especies Vulnerables que componen el Catálogo Valenciano de Especies de Flora Amenazadas (CVEFA) se conserva germoplasma en el banco de germoplasma del Servicio de Vida Silvestre en el CIEF, también denominado como colección CIEF del Banco de Germoplasma de Flora Silvestre Valenciana.

Para el 67,6\% de las unidades de seguimiento actualmente conocidas de las especies catalogadas como En Peligro y para el 50\% de las unidades de seguimiento de las especies Vulnerables, se conserva germoplasma en este banco.
La combinación de determinados parámetros de los que se dispone en la actualidad en la base de datos del banco de germoplasma permite relacionar información sobre la calidad del material recolectado y conservado con un origen geográfico, permitiendo asimismo obtener nueva información relevante acerca de posibles causas de amenaza de las especies y facilitando al mismo tiempo una planificación de fututo respecto a la intensidad y localización de nuevas recolecciones.

El análisis SIG de las accesiones georreferenciadas permite direccionar y focalizar el esfuerzo y el trabajo a zonas y poblaciones de especies de interés, lo que puede repercutir en una mejora del estado de conservación de las especies y el ahorro de recursos. 
Aguilella, A., Fos, S. \& Laguna, E. (eds.). 2009. Catálogo Valenciano de Especies de Flora Amenazadas. Colección Biodiversidad, 18. Conselleria de Medi Ambient, Aigua, Urbanisme i Habitatge, Generalitat Valenciana. Valencia. 358 pp.

Banco de Datos de Biodiversidad de la Comunidad Valenciana. Conselleria de Medi Ambient, Aigua, Urbanisme i Habitatge, Generalitat Valenciana. http://bdb.cth.gva.es/

Bacchetta G., Bueno-Sánchez A., Fenu G., Jiménez-Alfaro B., Mattana E., Piotto B. \& Virevaire M. (eds). 2008. Conservación ex situ de plantas silvestres. Principado de Asturias. La Caixa. 378 pp

Bañares, A. (ed.). 2003. Biología de la conservación de plantas amenazadas. Ministerio de Medio Ambiente. Madrid. $270 \mathrm{pp}$.

Beck, J., Ballesteros-Mejía, L., Nagel, P. \& Kitching, I.J. 2013. Online solutions and the "Wallacean shortfall": what does GBIF contribute to our knowledge of species' ranges? Divers. Distrib. 19(8): 1043-1050.

Boakes, E.H., Mcgowan, P.J.K., Fuller, R.A., Chang-Qing, D., Clark, N.E., O’Connor, K. \& Mace, G.M. 2010. Distorted views of biodiversity: spatial and temporal bias in species occurrence data. PLoS Biology 8(6): e1000385.

Cohen, J.I., Williams, J.T., Plucknett, D.L. \& Shands, H.L. 1991. Ex situ conservation of plant genetic resources: global development and environmental concerns. Science 253: 866-872.

Decreto 70/2009, de 22 de mayo, del Consell, por el que se crea y regula el Catálogo Valenciano de Especies de Flora Amenazadas y se regulan medidas adicionales de conserva-ción. Diari Oficial de la Comunitat Valenciana 6021: 20143-20162.

Falk, D.A. 1987. Integrated conservation strategies for endangered plants. Nat. Areas J. 7: 118-123.

Ferrer-Gallego, P.P., I. Ferrando, Escribá, M.C., Albert, F., Navarro, A., Martínez, V., Hurtado, A. \& Laguna, E. 2013a. El Banco de germoplasma de la flora silvestre valenciana: La colección CIEF (1990-2012). Chronica naturae 3: 76-82.

Ferrer-Gallego, P.P., Ferrando, I., Gago, C. \& Laguna, E. (eds.) 2013b. Manual para la conservación de germoplasma y el cultivo de la flora valenciana amenazada. Colección Manuales Técnicos Biodiversidad, 3. Conselleria d'Infraestructures, Territori i Medi Ambient. Generalitat Valenciana. Valencia. 252 pp.
Frew, J. E. \& Dozier, J. 2012. Environmental Informatics. Annu. Rev. Env. Resour. 37(1): 449-472.

Hamilton, M.B. 1994. Ex situ conservation of wild plant species: time to reassess the genetic assumptions and implications of seed banks. Conserv. Biol. 8: 39-49.

IPGRI/FAO/FLD 2004. Forest genetic resources conservation and management. Vol. 3. Plantations and genebanks. Biodiversity International. Rome.

Iriondo, J. M. 2001. Conservación de germoplasma de especies raras y amenazadas (Revisión). Invest. Agra. Prod. Veg. 16(1): 6-24.

Iriondo, J.M., Coord. 2011. Atlas y Libro Rojo de la Flora Vascular Amenazada de España. Manual de metodología corológico y demográfico. Dirección General de Medio Natural y Política Forestal (Ministerio de Medio Ambiente, y Medio Rural y Marino)-Sociedad Española de Biología de la conservación de Plantas. Madrid, 70 pp.

Kelling, S., Hochachka, W.M., Fink, D., Riedewald, M., Caruana, R., Ballard, G. \& Hooker, G. 2009. Dataintensive Science: A New Paradigm for Biodiversity Studies. BioScience 59(7): 613-620.

Laguna, E. 2002. Estrategia de conservación de la flora silvestre en la Comunidad Valenciana. Conserv. Vegetal 7: 12-13.

Laguna, E., Ferrer-Gallego, P.P., Escribá, M.C., Peña, C., Sebastián, A., I. Ferrando, Albert, F.J. \& Navarro, A. 2012. El efecto de la normativa de protección en la mejora de la conservación ex situ de especies amenazadas: germinación de plantas catalogadas en la Comunidad Valenciana (España). Cuadernos de Biodiversidad 40: 1-7.

Orden 6/2013, de 25 de marzo, de la Conselleria de Infraestructuras, Territorio y Medio Ambiente, por la que se modifican los listados valencianos de especies protegidas de flora y fauna. Diari Oficial de la Comunitat Valenciana 6996: 8682-8690.

Prada, M.A. Herreros, R. Ortiz, G. \& Marzo, M. 2012. ECOGEN. Programa Valenciano de Conservación de Recursos Genéticos Forestales. 2011-2020. Conselleria de Infraestructuras, Territorio y Medio Ambiente. Generalitat Valenciana.

Uribe, F. \& Prieto, M. 2014. Georreferenciar antiguas colecciones de Ciencias Naturales: de la artesanía a la técnica. Bol. R. Soc. Esp. Hist. Nat. Secc. Aula, Museos y Colecciones 1: 1-11. 